\title{
Orbital retinoblastoma: case report
}

\author{
Retinoblastoma orbitário: relato de caso
}

Eduardo Darahem Mabtum ${ }^{1}$, Maria Teresa Brizzi Chizzotti Bonanomi ${ }^{1}$, Patricia Picciarelli de Lima ${ }^{1}$, Maria Tereza Assis de Almeida ${ }^{1}$

\begin{abstract}
We describe the case of a 9-month old boy with unilateral retinoblastoma and bulftalmo. Primary enucleation was the treatment of choice due to the lack of visual prognosis. The histology of the enucleated eye showed massive choroidal invasion by the tumor and the optic nerve free of neoplastic tissue. Therefore, no adjuvant chemotherapy or radiotherapy was indicated. Three months after the enucleation, the patient returned with massive orbital retinoblastoma with exposure of the conjunctiva. Treated with chemotherapy, the patient has been in remission for 12 months. The risk factors for orbital recurrence are discussed.
\end{abstract}

Keywords: Orbital retinoblastoma; Risk factors; Recurrence/drug therapy; Chemotherapy, adjuvant; Case report

\section{RESUMO}

Relatamos paciente de 9 meses de idade com buftalmo e retinoblastoma unilateral. O paciente foi tratado com enucleação primária devido à falta de prognóstico visual. O exame do olho enucleado mostrou invasão maciça de coroide e ausência de comprometimento do nervo óptico não sendo, portanto, submetido a tratamento adjuvante de quimioterapia ou de radioterapia. Três meses após a enucleação, o paciente apresentou-se com retinoblastoma orbitário volumoso com exposição da conjuntiva. Tratado com quimioterapia permanece em remissão após 12 meses. São discutidos os fatores de risco para a ocorrência da recidiva orbitária.

Descritores: Retinoblastoma orbitário; Fatores de risco; Recorrência/quimioterapia Quimioterapia adjuvante; Relato de caso

\section{INTRODUCTION}

Retinoblastoma is an intraocular neuroectodermal neoplasia. Unilateral disease is the most common form, occurring in 60 to $70 \%$ of the cases $^{(1)}$. Between $4.2 \%$ and $9.5 \%$ of patients who undergo enucleation present orbital recurrence ${ }^{(2)}$, and the main risk factors are compromised surgical margins and extrascleral invasion ${ }^{(3,4)}$. The infiltration of the retrolaminar optic nerve, regardless of its association with choroidal invasion, is also associated with tumor recurrence, and an adjuvant treatment may be indicated. Conversely, isolated choroidal invasion as a risk factor is controversial. While some authors suggest adjuvant treatment ${ }^{(5)}$, others argue that choroidal involvement does not justify additional therapy ${ }^{(6)}$. We report a case of retinoblastoma with isolated choroidal invasion and orbital recurrence.

\section{CASE REPORT}

A 9-month-old boy was admitted to the Hospital das Clínicas da Faculdade de Medicina da Universidade de São Paulo on August 3, 2010 with leukocoria in the right eye (RE). On examination, he presented no vision and buphthalmia in the RE and normal fixation and no changes in the left eye. Ultrasonography and magnetic resonance imaging (MRI) of the RE showed an intraocular tumor presenting calcification compatible with retinoblastoma (Figure 1). The anatomopathological examination of the enucleated RE showed moderately differentiated retinoblastoma occupying the entire vitreous cavity with massive choroidal invasion and without optic nerve invasion (Figure 2). The patient returned after 3 months with massive proptosis and conjunctival exposure. The MRI finding was consistent with tumor recurrence (Figure 3), which was confirmed by biopsy. The patient was then subjected to 6 cycles of chemotherapy with vincristine sulfate, etoposide phosphate and carboplatin (VEC) and 45 Gy of external radiotherapy, with complete tumor reduction and no recurrence after 24 months.

\section{DISCUSSION}

Currently, retinoblastoma has been conservatively treated using various methods with excellent results. If the disease is at a very advanced stage and no vision recovery is expected, enucleation is indicated and is considered curative for the intraocular tumor. After the enucleation of an eye with many anatomical changes, such as those our patient presented, orbital recurrence is unpredictable, and knowledge of the risk factors is essential. Orbital tumor and retrolaminar nerve invasion are predictive of metastatic disease ${ }^{(2,4,5,7,8)}$, and retrolaminar optic nerve invasion, especially with compromised surgical margins and scleral invasion, are major risk factors for orbital recurrence ${ }^{(5,8)}$. In a study of 1,674 enucleated eyes, 71 (4.2\%) developed orbital recurrence; only 2 presented scleral erosion, 32 presented choroidal invasion, 35 presented postlaminar optic nerve invasion (7 with positive surgical margins), and 11 had no histological risk factor ${ }^{(2)}$.

Neovascular glaucoma could be a relative risk factor for the orbital extension of the tumor because the increase in IOP would cause thinning of the sclera and cornea, which would facilitate the tumor's extension ${ }^{(7)}$; meanwhile, the massive choroidal invasion would give the tumor access to the emissary veins and the sclera, especially in patients with glaucoma, and facilitate tumor metastasis ${ }^{(4,7)}$. Evidently, it is impossible to histologically detect all of the tumor cells that have

Submitted for publication: October 9, 2012

Accepted for publication: July 27, 2013

Study carried out at Ophthalmology Department, Hospital das Clínicas, Universidade de São Paulo - USP, São Paulo (SP), Brazil.

Physician, Ophthalmology Department, Hospital das Clínicas, Universidade de São Paulo - USP, São Paulo (SP), Brazil.

Funding: No specific financial support was available for this study.

Disclosure of potential conflicts of interest: E.D.Mabtum, None; M.T.B.C.Bonanomi, None; P.P.Lima, None. M.T.A.de Almeida, None.

Correspondence address: Eduardo Darahem Mabtum. Hospital das Clínicas da Faculdade de Medicina da USP - Departamento de Oftalmologia. Av. Dr. Enéas de Carvalho Aguiar, 255 - 6o andar São Paulo - SP - 05403-000 - Brazil - E-mail: emabtum@hotmail.com 

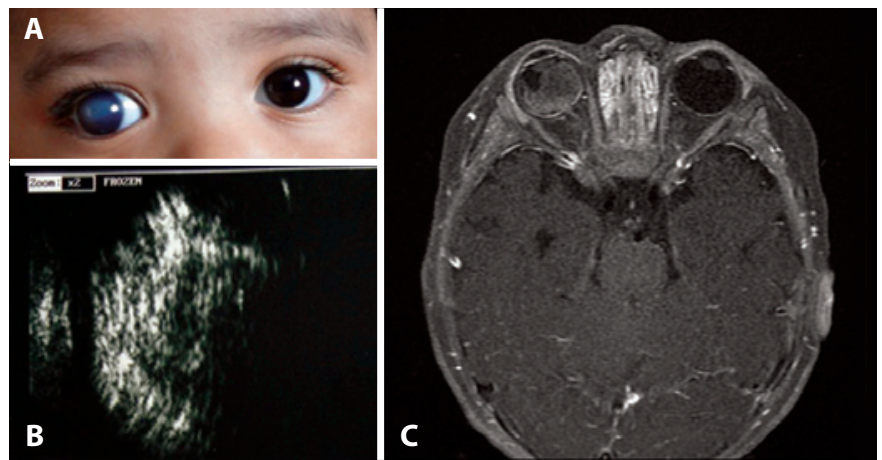

Figure 1. A) Clinical aspect of buphthalmia in the right eye. B) Ultrasonography demonstrated calcium within the tumor. $206 \times 127 \mathrm{~mm}(96 \times 96 \mathrm{dpi})$.C) Magnetic resonance image showing an intraocular tumor occupying the entire vitreous cavity without changing the posterior contour of the sclera in T1 (without extrascleral invasion suspicion).
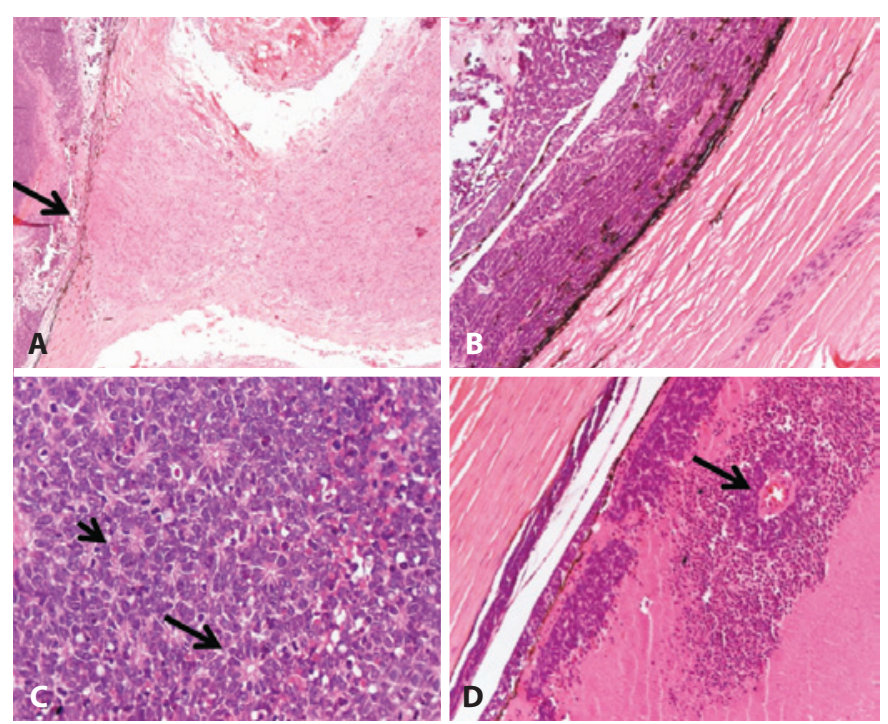

Figure 2. A) General view of the optic nerve, partially showing the lamina cribrosa (arrow) without tumor cell invasion. B) Massive choroidal invasion with tumor cells that infiltrate the entire thickness of this layer. C) Microscopy of the tumor tissue showing poorly differentiated neuroblastic cells, minimal cytoplasm, basophilic nuclei (several of them pyknotic), mitotic figures and Flexner-Wintersteiner (arrow) and Homer-Wright rosettes (arrowhead). D) Viable tumor cells invading the choroid, with a necrotic area and the presence of pseudorosettes with a central blood vessel (arrow). $203 \times 130 \mathrm{~mm}$ ( $96 \times 96 \mathrm{dpi}$ ).

left the intraocular area and lodged in the orbit. A retrospective study of 182 consecutive cases of enucleated unilateral retinoblastoma shows an association of glaucoma and buphthalmia with high-risk anatomopathological findings ${ }^{(9)}$. Our patient presented with neovascular glaucoma and buphthalmia, with massive choroidal invasion as the only risk factor identified upon anatomopathological analysis, which did not justify adjuvant treatment according to our protocol. Adjuvant therapy with chemotherapy in the postoperative period, regardless of whether it is associated with radiotherapy, is indicated when retrolaminar nerve invasion is present with or without choroidal invasion or in the presence of scleral invasion ${ }^{(3,10)}$. However, the importance of even massive isolated choroidal invasion is controversial. An analysis of 361 cases in the literature showed that only nerve and orbit invasions are predictive of death, and isolated choroidal invasion is not lethal(5). A retrospective analysis of 224 eyes enucleated by unilateral retinoblastoma showed that among 108 eyes with high-risk features, 55 had isolated choroidal invasion, and 16 of these

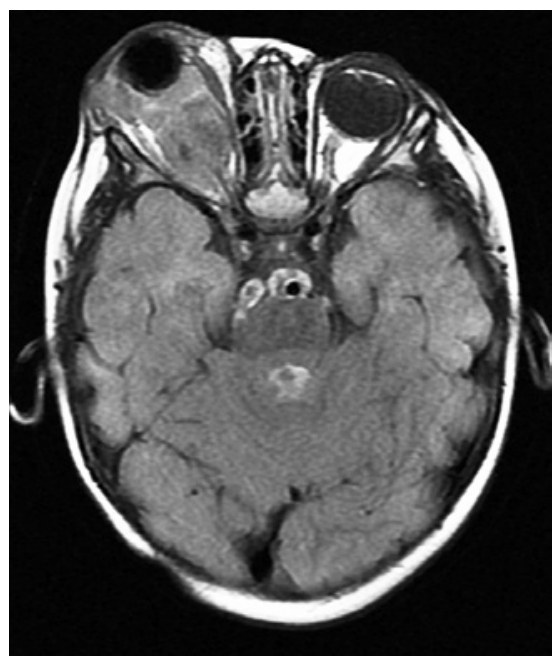

Figure 3. Magnetic resonance image three months after the enucleation, with a tumor mass occupying the entire orbit and moving the polymethylmethacrylate orbital implant out of the orbit. $203 \times 130 \mathrm{~mm}(96 \times 96 \mathrm{dpi})$.

had prelaminar nerve invasion. None of the patients underwent adjuvant treatment, and 3 had orbit recurrence, 2 of them with associated systemic involvement. Because all of the patients survived intensive chemotherapy, the authors concluded that the risk of the adjuvant treatment is unnecessary and may be avoided; they do not indicate adjuvant treatment for cases with isolated choroidal invasion ${ }^{(6)}$.

In contrast, we should remember that orbital retinoblastoma is associated with a 10 to 27 times greater risk of metastasis ${ }^{(3,5,8)}$, which can be devastating ${ }^{(11)}$. A prospective study of 80 enucleated eyes with unilateral retinoblastoma and high-risk histological features (anterior chamber, iris, ciliary body and massive choroidal, retrolaminar nerve and extrascleral invasions) divided the patients to groups of 46 patients with and 34 patients without adjuvant therapy after the enucleation. The study found a significant difference in the incidence of metastasis between the treated (4\%) and untreated group (23\%) among patients with massive choroidal invasion or retrolaminar optic nerve invasion without any important complications. The authors concluded that adjuvant chemotherapy is safe and effective and significantly reduces the occurrence of metastases in patients with high-risk histological features ${ }^{(12)}$.

The fact that our patient presented with a substantial orbital recurrence only 3 months after enucleation demonstrates that some tumor cells were already present in the extraocular areas when the enucleation was performed. In fact, orbital recurrence can occur 1 to 24 months after the enucleation. The majority (97\%) of recurrences occur in the first 12 months ${ }^{(2)}$ and can be treated successfully, as was the case for this patient, who remained disease-free 24 months after treatment.

If chemotherapy had been administered prior to the enucleation, could the recurrence have been avoided? A controversial retrospective study of 100 patients treated in Beijing stated that prior chemotherapy may increase deaths from metastasis because histological features of risk may be masked, which results in reduced monitoring and delayed treatment ${ }^{(13)}$. This concern was allayed by a multicenter consensus recommending that current protocols include preoperative chemotherapy followed by planned enucleation and continuing cycles after surgery. This preoperative treatment is helpful for patients with MRIs that suggest extraocular disease or patients with clinically suspicious conditions, such as proptosis and buphthalmia ${ }^{(14)}$.

Unilateral retinoblastoma associated with glaucoma and buphthalmia must be subjected to chemotherapy with therapeutic doses 
of VEC and should never receive subtherapeutic doses. Enucleation can be safely planned between the second and the fourth cycle, and the treatment should occur until the sixth cycle, as for intraocular retinoblastoma treatment ${ }^{(14)}$. Alternatively, the same systemic treatment can be used after the detection of massive isolated choroidal invasion because the VEC multidrug protocol proved to be more effective at preventing metastases ${ }^{(15)}$.

\section{REFERENCES}

1. Bonanomi MT, Almeida MT, Cristofani LM, Odone Filho V. Retinoblastoma: a three year-study at a Brazilian medical school hospital. Clinics. 2009;64(5):427-34

2. Kim JW, Kathpalia V, Dunkel IJ, Wong RK, Riedel E, Abramson DH. Orbital recurrence of retinoblastoma following enucleation. Br J Ophthalmol. 2009;93(4):463-67.

3. Khelfaoui F, Validire P, Auperin A, Quintana E, Michon J, Pacquement H, et al. Histopathologic risk factors in retinoblastoma: a retrospective study of 172 patients treated in a single institution. Cancer. 1996;77(6):1206-13.

4. Messmer EP, Heinrich T, Höpping W, Sutter E, Havers W, Sauerwein W. Risk factors for metastases in patients with retinoblastoma. Ophthalmology. 1991;98(2):136-41.

5. Kopelman JE, McLean IW, Rosenberg SH. Multivariate analysis of risk factors for metastasis in retinoblastoma treated by enucleation. Ophthalmology. 1987;94(4): 371-77.

6. Chantada GL, Dunkel IJ, de Dávila MT, Abramson DH. Retinoblastoma patients with high risk ocular pathological features: who needs adjuvant therapy? Br J Ophthalmol. 2004;88(8):1069-73.

7. Shields CL, Shields JA, Baez KA, Cater J, Potter PV. Choroidal invasion of retinoblastoma: metastatic potential and clinical risk factors. Br J Ophthalmol. 1993;77(9):544-8.

8. Singh AD, Shields CL, Shields JA. Prognostic factors in retinoblastoma. J Pediatr Ophthalmol Strabismus. 2000;37(3):134-41.

9. Chantada GL, Gonzalez A, Fandino A, de Davila MT, Demirdjian G, Scopinaro M, et al. Some clinical findings at presentation can predict high-risk pathology features in unilateral retinoblastoma. J Pediatr Hematol Oncol. 2009;31(5):325-9.

10. Gündüz K, Müftüoglu O, Günalp I, Unal E, Taçyildiz N. Metastatic retinoblastoma clinical features, treatment, and prognosis. Ophthalmology. 2006;113(9):1558-66.

11. Antoneli CB, Steinhorst F, de Cássia Braga Ribeiro K, Novaes PE, Chojniak MM, Arias V, et al. Extraocular retinoblastoma: a 13-year experience. Cancer. 2003;98(6):1292-8.

12. Honavar SG, Singh AD. Management of advanced retinoblastoma. Ophthalmol Clin North Am. 2005;18(1):65-73.

13. Zhao J, Dimaras H, Massey C, Xu X, Huang D, Li B, et al. Pre-enucleation chemotherapy for eyes severely affected by retinoblastoma masks risk of tumor extension and increases death from metastasis. J Clin Oncol. 2011;29(7):845-51.

14. Chantada G, Leal-Leal C, Brisse H, de Graaf P, Sitorus RS, Qaddoumi I, et al. Is it preenucleation chemotherapy or delayed enucleation of severely involved eyes with intraocular retinoblastoma that risks extraocular dissemination and death? J Clin Oncol. 2011:29(24):3333-4.

15. Kaliki S, Shields CL, Rojanaporn D, Al-Dahmash S, McLaughlin JP, Shields JA, et al. High-risk retinoblastoma based on international classification of retinoblastoma: analysis of 519 enucleated eyes. Ophthalmology. 2013;120(5):997-1003. 